\title{
Nondestructive Testing of Marine Protective Coatings Using Terahertz Waves with Stationary Wavelet Transform
}

\author{
Wanli Tu${ }^{1,2}$, Shuncong Zhong ${ }^{1,3^{*}}$, Yaochun Shen ${ }^{1,4}$, and Atilla Incecik ${ }^{3}$ \\ ${ }^{1}$ Laboratory of Optics, Terahertz and Non-destructive Testing, School of Mechanical Engineering and Automation, \\ Fuzhou University, Fuzhou, 350108, P.R. China \\ ${ }^{2}$ Marine Engineering Institute, Jimei University, Xiamen, 361021, P.R. China \\ ${ }^{3}$ Department of Naval Architecture, Ocean and Marine Engineering, University of Strathclyde, Glasgow G4 OLZ, United Kingdom \\ ${ }^{4}$ Department of Electrical Engineering and Electronics, University of Liverpool, Liverpool L69 3GJ, United Kingdom \\ *Corresponding author:zhongshuncong@hotmail.com
}

\begin{abstract}
Terahertz wave propagation in marine protective coatings and its non-destructive testing (NDT) capability were studied by the finite difference time domain (FDTD) method. The FDTD model was used to calculate the propagation and reflection of $\mathrm{THz}$ radiation from marine protective coatings. The reflected terahertz waves could be employed in coating thickness analysis of the paint layers. In order to clearly identify the interface between antifouling and anticorrosive coatings, Stationary Wavelet Transform (SWT) approach was applied to decompose the obtained terahertz impulse functions into approximation and detail coefficients; SWT detail coefficients were used for the feature extraction of the coating thickness. SWT provides a more accurate identification of salient features in a signal, such as the weak feature between antifouling and anticorrosive coatings. We found that the developed model and SWT-based algorithms could be used to evaluate the occurrence of defects beneath the coatings (e. g., paint-off and corrosion defects). The proposed method provides the solution for coating thickness of marine protective coatings and it would benefit the effective maintenance to avoid coating failure and facilitate marine protective coating design. Therefore, nondestructive testing and evaluation of marine protective coating system by terahertz waves with SWT could be recommended for engineering applications.
\end{abstract}

Keywords: Marine protective coatings, Non-destructive testing, Stationary wavelet transform, Terahertz wave, Finite difference time domain 


\section{Introduction}

Protective coating is usually applied for marine structures and offshore plants as a corrosion protection. However, deterioration, aging or failure occurs in the harsh marine environment, especially the corrosion deterioration. During the corrosive process, the chemical and physical characteristics changes in the coating system and thus forms various defects, such as bubbling, rust, cracking, shedding etc. [1-4]. Severe corrosion degradation may lead to the replacement of a complete ship panel (deck, side, bottom, etc.). Thus the monitoring of the degradation of paint layers is of great importance for the long term use of the marine protective coatings [5]. In addition to general (uniform) corrosion which reduces the plate thickness uniformly, there are other types of more localized corrosion, such as corrosion, pitting, and detachment, blistering, etc. A fast, reliable detection method to detect possible coating defects and to evaluate coating performance can provide timely and effective maintenance to avoid serious consequences due to coating failure. Corrosion processes beneath organic coatings are usually monitored by using conventional electrochemical techniques, such as electrochemical impedance spectroscopy (EIS), electrochemical noise measurements (ENM) and so on, which allow both the evaluation of corrosion rates and the identification of corrosion mechanisms [6-9]. EIS uses an external voltage or current source; for this reason, corrosion processes can be disturbed, and the results may not be perfectly reliable. Furthermore, it can only provide the assessment of uniform corrosion of interface, which was difficult to give the local corrosion inspection [10]. The application of ENM method does not involve the artificial disturbance of the system and data analysis could be performed in the electrochemical noise records to capture information about the type of corrosion damage. However, because of the very small voltages and currents involved, most systems are characterized by a high number of overlapped transients, thus ENM data can sometimes be affected by extraneous signals (although normally the results are changed by only a factor of two or less) which make it need to be performed using adequate mathematical tools and not so suitable for monitoring or quality control [11-12]. Other commercially available thickness measurement techniques for protective 
coatings including ultrasonic testing [13] and eddy-current testing [14] etc. Furthermore, some optical probing methods such as eddy current pulsed thermography [15] and infrared thermography [16] are also adopted for the defect measurement of paint film.

Time Domain Terahertz (TD-THz) technology provides the capability for standoff inspection of coatings that are otherwise opaque and cause strong scattering at visible and infrared wavelengths. Recent advances in TD-THz technology has been extensively used in the sensing and imaging fields because of its many merits such as non-contact, non-destructive and that many materials are relatively transparent to $\mathrm{THz}$ waves [17]. Besides, the photon energy of terahertz radiation is in the range of a few meV (one thousand times smaller than that of UV light and one million times smaller than that of X-ray)[17], which makes TPI technology much safer than X-ray testing although X-ray can give high-precision and accurate measurement, regarding to the safe operating procedures[18]. When a terahertz pulse is incident on a multilayered medium, the reflected or transmitted signals would record information whenever there is a change in the refractive index or the optical absorption coefficient of the material (caused by either chemical or structural changes in the medium). The structural information can be ultimately extracted by analysis of the recorded terahertz wave in the time domain. So far, terahertz pulsed imaging (TPI) has become an established powerful non-destructive testing tool to characterize a range of multilayered samples, for example, pharmaceutical solid dosage forms [19], dental tissues [20], layered polymer composites [21] and other extensive investigation for industrial application such as detecting materials under coating layers [22-25], detecting defects within the sprayed on foam insulation [26], and detecting corrosion under metallic source material [27]. It showed that for thick samples such as the multilayer medium above, the detected echoes of the terahertz signal, which are caused by multiple reflections in the sample, can be time separated well.

To our best knowledge, there were some researches about TPI technique testing for protective coatings. The utility of time-domain terahertz technology for automobile paint thickness measurements has 
previously been demonstrated in the laboratory by T. Yasui et al. [28-29]. T. Fukuchi et al. determined refractive index and thickness of the topcoat of a thermal barrier coating from the reflected waveforms of terahertz waves [30]. T. Kurabayashi and coworkers studied corrosion detection under paint films using a specific frequency of $\mathrm{THz}$-wave in $\mathrm{THz}$ transmitted imaging system. They found the mechanical defects and thru holes were visualized, but corrosion areas were not [31]. David J. Cook and coworkers discussed the applicability of terahertz non-destructive testing for marine protective coatings and took a laboratory investigation to measure the dry film thickness of organic coatings. They were developing a standoff sensor for the real-time thickness measurement of wet (uncured) marine paints for the purpose of providing feedback to an automated system for painting ships in dry-dock [32-33]. The research showed that TPI technology could be an excellent complimentary means to the protective coating testing.

So far, to our best knowledge, the above researches on TPI technique testing for protective coatings are focused on experimental aspects. However, there were few theoretical studies about terahertz radiation interacts with protective coatings numerically and theoretically. Advanced numerical modeling and further theoretical analysis of the interaction of terahertz radiation with marine protective coatings will be extremely valuable for the better application of TPI technology in ship, especially for rapid hidden defect detection(such as peeling, blistering and corrosion, etc.) and quantitative analysis, which will provide timely and reliable information for ship maintenance work. Indeed, some theoretical analysis have already been reported to study the propagation of terahertz radiation in various multilayered media. For terahertz pulsed imaging in which a transient terahertz pulse is used, a time-domain method, such as the finite difference time domain (FDTD) method, is more applicable than a frequency-domain method because a differential equation is simpler to solve than an integral equation. The FDTD algorithm is a proven numerical method to model electromagnetic scattering problems, which solves the Maxwell's equations directly and obtains the solution of the electric field of electromagnetic waves [34]. It analyses continuous electromagnetic problems by using finite difference and obtains the electric field value at the sampling point. The FDTD method offers several 
advantages, such as robustness and the ability to study dispersive, nonlinear, or anisotropic materials [35-36]. In our previous works, a theoretical model based on the FDTD method for pharmaceutical-coated tablets was investigated and the simulated results were found to be in good agreement with the experimental results [3738]. It has been demonstrated that FDTD- model could be capable of calculating the propagation and reflection of $\mathrm{THz}$ radiation from a multilayered flat or curved structure.

In the present work, non-destructive testing of marine protective coatings was investigated by TPI technology with reflection-type detection mode using FDTD method combined with stationary wavelet transform approach. A FDTD-based three-dimensional (3D) model used to calculate and analyze the reflected THz wave from marine protective coatings was proposed. The numerical modeling and theoretical analysis of the interaction of terahertz radiation with marine protective coatings was investigated. This is not only valuable for the better application of TPI technology in marine protective coatings, but also for analysis of other non-metallic coatings on metal substrate. Current TPI technique provides few quantitative information (such as the thickness of paint layer) and possible defect testing of marine protective coatings, especially for the paint-off and corrosion defects which need to be detected when the ship was under period maintenance. We carried out theoretical analysis of coating thickness analysis for each paint layer of marine protective coatings and non-destructively evaluation of the occurrence of defects in the coatings. Different protective coating systems with different paint layer thickness were modeled and computed using the FDTD method with the broad-band terahertz radiation. Besides, multilayered samples to mimic the occurrence of various defects (paint-off defect and corrosion defect) in coating were investigated. Furthermore, stationary wavelet transform approach was used to terahertz impulse functions for quantitative coating analysis and defect identification of marine protective coatings.

\section{FDTD Modeling of Terahertz Wave Propagation in Marine Protective Coatings}


FDTD is a direct solution of Maxwell's time-dependent curl equations. It applies simple, second-order accurate central-difference approximations for the space and time derivatives of the electric and magnetic field directly to the differential operators of the curl equations. In a 3D Cartesian coordinate system, the time-dependent Maxwell's curl equations are arranged in the following form as [39]:

$$
\begin{aligned}
& \frac{\partial E_{x}}{\partial t}=\frac{1}{\varepsilon}\left(\frac{\partial H_{z}}{\partial y}-\frac{\partial H_{y}}{\partial z}-\sigma E_{x}\right) \\
& \frac{\partial E_{y}}{\partial t}=\frac{1}{\varepsilon}\left(\frac{\partial H_{x}}{\partial z}-\frac{\partial H_{z}}{\partial x}-\sigma E_{y}\right) \\
& \frac{\partial E_{z}}{\partial t}=\frac{1}{\varepsilon}\left(\frac{\partial H_{y}}{\partial x}-\frac{\partial H_{x}}{\partial y}-\sigma E_{z}\right) \\
& \frac{\partial H_{x}}{\partial t}=-\frac{1}{\mu}\left(\frac{\partial E_{z}}{\partial y}-\frac{\partial E_{y}}{\partial z}\right) \\
& \frac{\partial H_{y}}{\partial t}=-\frac{1}{\mu}\left(\frac{\partial E_{x}}{\partial z}-\frac{\partial E_{z}}{\partial x}\right) \\
& \frac{\partial H_{z}}{\partial t}=-\frac{1}{\mu}\left(\frac{\partial E_{y}}{\partial x}-\frac{\partial E_{x}}{\partial y}\right)
\end{aligned}
$$

where $E x, E y, E z$ are components of electric field, $H x, H y, H z$ are components of magnetic field, $\varepsilon$ is the electric permittivity, $\sigma$ is the electric conductivity, $\mu$ is the magnetic permeability and equivalent magnetic loss is ignored. With FDTD method, the model space is divided into small cubes called Yee cells by grid, with a grid size $\Delta x=\Delta y=\Delta z=\delta$ less than the size of relevant features, and the time is divided into small steps (time step $\Delta t$ ) that are much less than the period of the relevant electromagnetic wave.

On the other hand, the central difference approximations are defined as

$$
\begin{aligned}
& \frac{\partial F^{n}(i, j, k)}{\partial x}=\frac{F^{n}\left(i+\frac{1}{2}, j, k\right)-F^{n}\left(i-\frac{1}{2}, j, k\right)}{\Delta x}+\operatorname{order}\left(\Delta x^{2}\right) \\
& \frac{\partial F^{n}(i, j, k)}{\partial t}=\frac{F^{n+\frac{1}{2}}(i, j, k)-F^{n-\frac{1}{2}}(i, j, k)}{\Delta t}+\operatorname{order}\left(\Delta t^{2}\right)
\end{aligned}
$$

where $i, j, k$ represent the nodes of the Yee cells, $n$ represents the calculated time step, $(i, j, k)=$ $(i \Delta x, j \Delta y, k \Delta z)$, and $F^{n}(i, j, k)=F(i \Delta x, j \Delta y, k \Delta z, n \Delta t)$. 
Upon substituting the central-difference approximations (7) (8) for the space and time derivatives into Maxell's equations, six couple finite-difference time-stepping expressions arise for the electric field components which were located on the three edges at each Yee cell, and magnetic field components which were located in the middle of the three surfaces. These expressions permit a progressive time integration of the Maxwell's equations suitable for the solution of an initial-value problem. The following is a sample time-stepping expression for an electric field component and the other components follow by analogy and can be found in our previous article [38].

$$
\begin{aligned}
& \left(1+\frac{\Delta t \cdot \sigma}{2 \varepsilon}\right)\left(E_{x}\right)_{\left(i+\frac{1}{2}, j, k\right)}^{n+1} \\
& \quad=\left(1-\frac{\Delta t \cdot \sigma}{2 \varepsilon}\right)\left(E_{x}\right)_{\left(i+\frac{1}{2}, j, k\right)}^{n}+\frac{\Delta t}{\delta \varepsilon}\left[\left(H_{z}\right)_{\left(i+\frac{1}{2}, j+\frac{1}{2}, k\right)}^{n+\frac{1}{2}}-\left(H_{z}\right)_{\left(i+\frac{1}{2}, j-\frac{1}{2}, k\right)}^{n+\frac{1}{2}}-\left(H_{y}\right)_{\left(i+\frac{1}{2}, j, k+\frac{1}{2}\right)}^{n+\frac{1}{2}}+\left(H_{y}\right)_{\left(i+\frac{1}{2}, j, k-\frac{1}{2}\right)}^{n+\frac{1}{2}}\right]
\end{aligned}
$$

Space and time discretizations are selected to bound errors in the sampling process and to insure numerical stability of the algorithm. With FDTD method, the stability criterion is of the form [39]

$$
\Delta t \leq 1 /\left(v_{\max } \sqrt{\frac{1}{\Delta x^{2}}+\frac{1}{\Delta y^{2}}+\frac{1}{\Delta z^{2}}}\right)
$$

where $v_{\max }$ is maximum wave speed in the material. In the model of our paper, it would be the light's velocity in the vacuum c. For the grid size $\delta=\Delta x=\Delta y=\Delta z$, the following equation was obtained:

$$
\Delta t \leq \delta / \sqrt{3} \mathrm{c}
$$

On the other hand, according to the reference [39], the following equation was chosen to satisfy for controlling numerical dispersion,

$$
\delta \leq \lambda / 10
$$

where $\lambda$ is the wavelength in the considered material. Furthermore, in order to remove the reflections from the back boundary, absorbing boundary conditions should be imposed [34]. 
Fig. 1 showed the simulation model of a terahertz pulse interacts with paint layers on a reflective (i.e., metal) substrate in reflection-type detection mode. The THz plane wave at an elevation angle theta and an azimuthal angle phi, is incident on the sample surface which is set at the $O x y$ plane. The azimuthal angle phi is referenced from the $x$ axis and the elevation angle theta is referenced from the $z$ axis. The FDTDbased three-dimensional model can be used for any type of input signal, which will be either a Gaussian pulse or a Sine wave. We chose a Gaussian pulse in the model because it has the advantage over the use of a Sine wave and it contains a wide band of frequencies. The reflections from the air/coating interface, internal interfaces existing at different paint layers and the coating/steel interface are indicated. The chemical and structural information in the medium would be encoded into the reflected $\mathrm{THz}$ waveform because it causes changes in the refractive index or the optical absorption coefficient of paint layers. As a result, the structural information of a sample can be ultimately extracted by analysis of the recorded $\mathrm{THz}$ wave in the time domain.

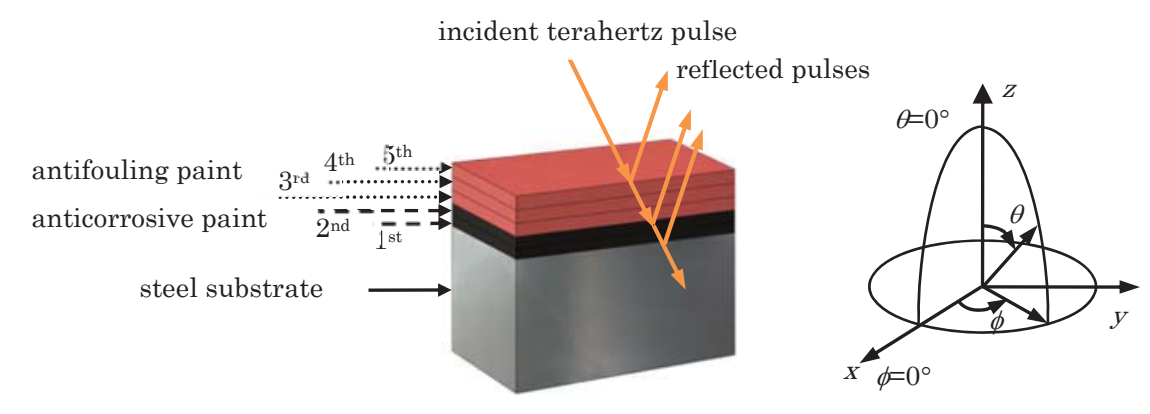

Fig. 1. Theoretical model of terahertz wave propagation in marine protective coatings

Generally, different areas of the ship require specific anti-corrosive coating. Steel corrosion at and around the waterline is of considerable practical interest for ships. Higher corrosion often occurs below the waterline attributed to the sewage pollution and undesirable accumulation of microorganisms, plants, and animals, which would accelerate bacterial activity in that region of the steel ship hulls. On the other hand, fouling is the attachment and growth of marine organisms on immersed surfaces and will raise the power requirements of ships and thus their fuel consumptions [40]. Therefore the coating system in that region is 
required to prevent corrosion and fouling, which is achieved by using an anticorrosive paint and an antifouling paint. The specific surface treatment method involves the application of organic coatings that exhibit high cavitation resistance and good erosion resistance [41], thus most paints are based on organic solvents. According to the reference [33], to mimic the marine protective coating below the waterline and the painting order, the coating system modeled in this paper contained: $1^{\text {st }}$ and $2^{\text {nd }}$ layer were anticorrosion paint layers, $3^{\text {rd }}, 4^{\text {th }}$ and $5^{\text {th }}$ were antifouling paint layers, as shown in Fig. 1.

\section{Stationary Wavelet Transform of Terahertz Impulse Functions}

The stationary wavelet transform (SWT) is a wavelet transform algorithm designed to overcome the lack of translation invariance of the discrete wavelet transform (DWT). Translation- invariance is achieved by removing the down samplers and up samplers in the DWT and up sampling the filter coefficients, which makes SWT as a redundant transform and provides a more accurate estimate of the variances at each scale and facilitates the identification of salient features in a signal, especially for recognizing noise or signal mutations. The SWT of the original data is not decimated, that is the size of the SWT data does not diminish after the transform, also known as undecimated wavelet transform [42-43]. The methodology based on SWT decomposition of terahertz impulse function into approximation coefficients $\left(\tilde{c}_{j+1, k}\right)$ and detail coefficients $\left(\tilde{d}_{j+1, k}\right)$ can be represented as follows:

$$
\begin{aligned}
\tilde{c}_{j+1, k} & =\left\langle f(x), \phi\left(\frac{x-k}{2^{(j+1)}}\right) / 2^{\frac{(j+1)}{2}}\right\rangle=\sum_{l=-\infty}^{+\infty} h(l) \tilde{c}_{j, k+2^{j} l} \\
\tilde{d}_{j+1, k} & =\sum_{l=-\infty}^{+\infty} h(l) \tilde{d}_{j, k+2^{j} l}
\end{aligned}
$$

where $f(x)$ is the deconvoluted result of THz detected signal, $\tilde{c}_{j, k}=\left\langle f(x), \phi\left(\frac{x-k}{2^{j}}\right) / 2^{\frac{j}{2}}\right\rangle$,

$\tilde{d}_{j, k}=\left\langle f(x), \psi\left(\frac{x-k}{2^{j}}\right) / 2^{\frac{j}{2}}\right\rangle$ are the approximation coefficients and detail coefficients, respectively, integer $j$ is decomposition step, $\phi(x)$ is the scaling function, $\psi(x)$ is the mother wavelet and $h$ is the impulse response of low-pass paraunitary quadrature mirror filters [44]. The more detail on SWT can be founded in ref [44]. 
Fig.2 shows a typical 3-level stationary wavelet transform decomposition of terahertz impulse function. $\mathrm{c} 1, \mathrm{c} 2$, and $\mathrm{c} 3$ are the SWT approximation coefficients for decomposition level of 1, 2, and 3 whist d1, d2, and $\mathrm{d} 3$ are the SWT detail coefficients for decomposition level of 1, 2 and 3. An important point to be clarified is the difference between the SWT application in the field of image processing and the field of signal processing of $\mathrm{THz}$ impulse function discussed here. In the field of image processing, an image signal is first decomposed by SWT, and then the detail coefficients are threshold by the thresholding rule to obtain a new detail coefficient. This new de-noised detail coefficient is then reconstructed with the approximation coefficient to produce a new image signal, which is a signal filtered by wavelet transform to enhance the image quality [44]. However, the SWT procedure in THz impulse function only involved one step of decomposition. THz impulse function was decomposed by SWT into approximation and detail coefficient. The detail coefficient provided information for coating thickness analysis and internal defect characterization. The thresholding and signal reconstruction were not needed in the terahertz non-destructive testing application for marine protective coatings.

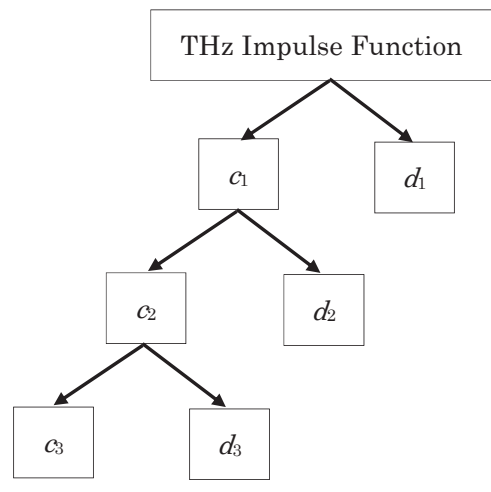

Fig. 2. Staionary wavelet transform decomposition of terahertz impulse function

\section{Results and Discussions}

\subsection{Thickness Coating Analysis of Marine Protective Coatings}

All FDTD simulations reported here are performed using the Remcom XFDTD commercial software package. In our model, the protective coating is treated as a linear isotropic homogeneous layer without 
magnetic field loss. For simplicity, the dispersive properties and the specific conductivity of coatings are negligible [33]. The parameters of the coatings were obtained from reference [33] which took an experimental investigation to measure the dry film thickness of organic coatings of marine protective coatings. The sample was taken as the simulated model in our studies and the paint thickness was set as theoretical thickness. A cylinder plate with thickness of $2 \mathrm{~mm}$ and radius of $20 \mathrm{~mm}$ was taken as steel substrate. To mimic the coating system below the waterline, two $152 \mu \mathrm{m}$ thick layers of anticorrosive paint (Amercoat 235, whose refractive index is 1.74) and three additional $127 \mu \mathrm{m}$ layers of antifouling paint (Interspeed 640, whose refractive index is 1.87) are successively coated on the steel substrate, as shown in Fig.1. The incident terahertz plane wave was set perpendicular to the sample surface (theta $=0^{\circ}, \mathrm{phi}=0^{\circ}$ ). A Gaussian profile of the amplitude distribution of the E-field was initiated in the incident plane with a peak amplitude of $1 \mathrm{~V} / \mathrm{m}$ and with a spectral coverage range covering $0.1 \mathrm{THz} \sim 1 \mathrm{THz}$ and pulse width was 34 time step. According to the stability criteria of the FDTD algorithm (as equations (11) and (12)), the simulation volume was divided into $0.03 \mathrm{~mm}$ by $0.03 \mathrm{~mm}$ by $0.03 \mathrm{~mm}$ Yee cells and the time step was determined as $\Delta t=0.0577 \mathrm{ps}$. According to the reference [45], the minimum detected thickness, that is the achievable depth resolution, is constrained to behalf the coherent length of the THz pulse in a sample. With a bandwidth of $\sim 1 \mathrm{THz}$, the corresponding coherence length in free space is about $\sim 100$ um [46]. The FDTD program was run for 4096 time steps with 8 perfectly matched layer (PML) absorbing boundary condition to remove the reflections from the back boundary. The far zone sensor and the planar sensor were employed to collecting the far-zone scatted field and the near-zone scattered fields.

The far-zone scatted field of object was often useful. In this simulated model, the far zone sensor was set at the position theta $=0^{\circ}$ and $\mathrm{phi}=0^{\circ}$, which is responsible for collecting the electric fields of the reflected pulses caused by the interfaces existed between different media, similar to the reflection mode of a TPI system, and the results represented the raw terahertz detected signals and were used in the deconvolution processing to get the impulse functions. Fig. 3 showed the FDTD results of the raw terahertz signal of coated 
steel substrates, which were collected by the far zone sensor. The sample for Fig.3 (a) was a two $152 \mu \mathrm{m}$ layers of anticorrosive paint coated on steel substrate whose material is Amercoat 235 with a refractive index of 1.74, whilst the one for Fig.3 (b) was a three $127 \mu \mathrm{m}$ antifouling paint layers whose material is Interspeed 640 with a refractive index of 1.87 plus the above two $152 \mu \mathrm{m}$ anticorrosive paint layers coated on steel substrate. In order to mimic the real experiments, Gaussian white noise was added to the terahertz pulse resulting in a SNR of $64 \mathrm{~dB}$ [47]. As can be seen from Fig.3 (b), due to large differences of refractive indices (RIs) of air, coating and steel, reflections from air/coating surface and the coating/steel interface can be clearly resolved. However, the reflection associated with internal interface (antifouling/anticorrosive paint interface) was very small because the RIs of antifouling and anticorrosive paint are similar. There was no reflection from the three antifouling paint layers and also from two anticorrosive paint layers because the RIs are same for the layers with same materials. The oscillation after the strong reflection from the coating/steel interface was caused by multiple reflections inside the sample [48].
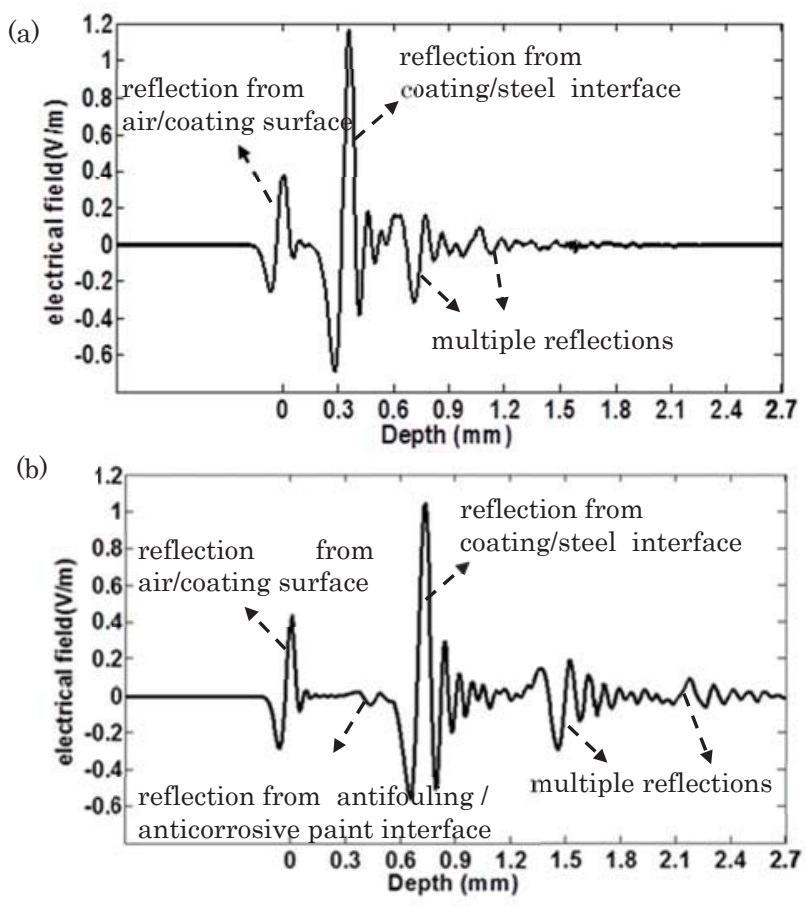

Fig. 3. Raw terahertz signal from coated steel substrates, which were collected by the far zone sensor from two samples: (a) two $152 \mu \mathrm{m}$ anticorrosive paint layers coated on steel substrate; (b) three $127 \mu \mathrm{m}$ antifouling paint layers plus two $152 \mu \mathrm{m}$ anticorrosive paint layers coated on steel substrate. 
In commercial software FDTD, the planar sensor is one of near-zone sensors, and it defines an entire plane (within the boundaries of the simulation space) to collect the data at the space grid note in the FDTD algorithm directly. The planar sensor is used to record time-varied electric and magnetic fields during the whole calculation period. Generally, the planar sensor can be set at cross section of the sample. Fig. 4 showed the FDTD simulation results (Fig.4 (a-2) (b-2)) collected by the planar sensor from the marine protective coating models. The corresponding thickness profiles (Fig4. (a-1) (b-1)). The colored regions represent the reflected waveforms caused by the inner interfaces between two different materials of the coating. The color in each figure represents the strength of the reflected signals. Dashed arrows indicate reflected terahertz waveform at the air/coating surface; solid arrows indicate the reflected waveform at the interface between protective coating and steel substrate; dotted arrow indicates the reflected waveform at the interface between antifouling paint and anticorrosive paint. As can be seen from the figure, the reflected waveforms caused by the interfaces can be recorded and identified. Also, there are some distortions at the edge of the sample. This is because scattering losses lead to significant distortion in the reflected terahertz pulse. Hence, the waveforms close to sharp edges are routinely removed before quantitative coating analysis [49]. Furthermore, as shown in Fig.4, the reflected waveform caused by antifouling/anticorrosive paint interface (dotted arrow in the Fig. 4(b-2)) was very weak due to the similar RIs of these two materials, which is match the raw detected signal obtained by far zone sensor shown as Fig.3(b). 


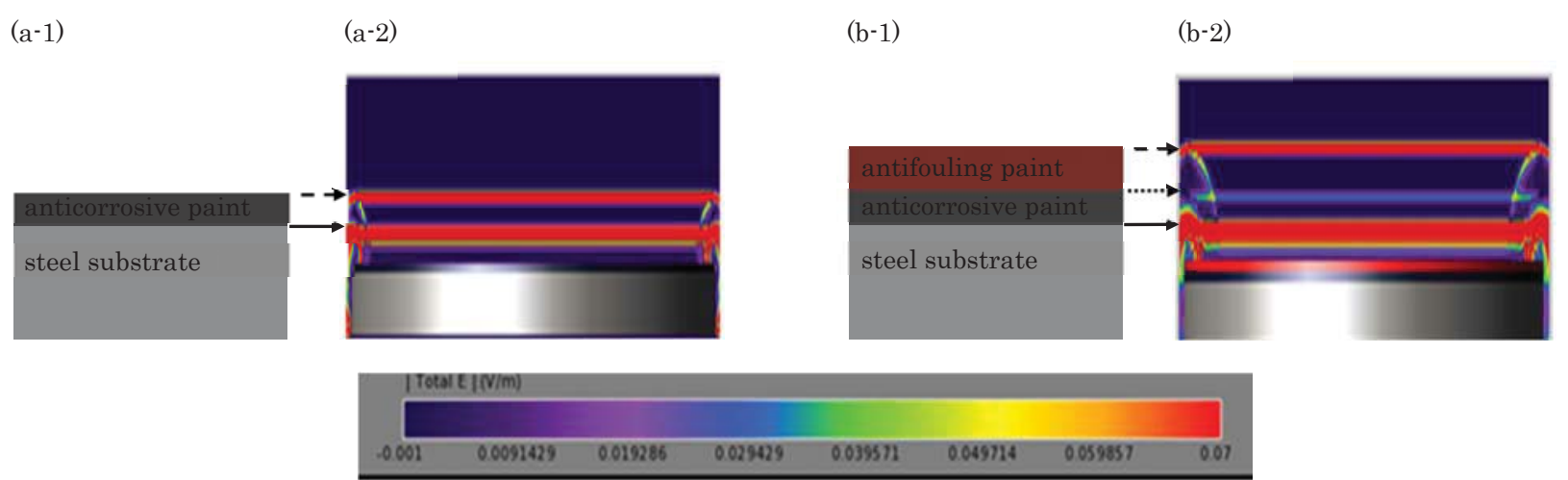

Fig. 4. The thickness profiles (a-1), (b-1) and FDTD simulation results (a-2), (b-2) for different marine protective coating models, which was collected by the planar sensor from two samples. (a) two $152 \mu \mathrm{m}$ anticorrosive paint layers coated on steel substrate; (b) three $127 \mu \mathrm{m}$ antifouling paint layers plus two $152 \mu \mathrm{m}$ anticorrosive paint layers coated on steel substrate. The colored regions represent the reflected waveforms caused by the inner interfaces between two different materials of the coating. The color stripe in each figure represents the strength of the reflected signals. Dashed arrows indicate reflected terahertz waveform at the air/coating surface; solid arrows indicate the reflected waveform at the interface between protective coating and steel substrate; dotted arrow indicates the reflected waveform caused by antifouling/anticorrosive paint interface.

In order to implement coating analysis and quantitative non-destructive evaluation (QNDE) of multilayer structures, terahertz impulse function is generally used. The impulse functions can be obtained in the frequency domain by deconvolution process, in which the raw terahertz waveform reflected off a coated structure is divided by the reference signal from a mirror [50]. In our model, the reference signal was collected from the reflections from a metallic surface. After deconvolution, the SWT approach was employed on the corresponding impulse function and, therefore, its detail coefficients were used for the coating thickness analysis.

Generally, the paint layers are successively coated on the steel substrate; also after each paint layer has been sprayed it need to be tested in the painting process. Thus, in order to mimic the painting process, we did a new FDTD simulation when a new paint layer was added since the painting worker will measure every paint layer coated. Fig.5 (a) and (e) showed normalized terahertz impulse functions (Fig.5 (a-1) (e-1)), the 
corresponding wavelet decomposition approximation coefficients (Fig.5 (a-2) (e-2)) and detail coefficients (Fig.5 (a-3) (e-3)) of different coated layers described above. When doing the deconvolution, a Gaussian band-pass filter was applied to remove high- and low- frequency components and also to produce a suitable time-domain response. A db2 wavelet was used to get appropriate time-frequency domain results in the SWT decomposition. As shown in Fig.5, the reflections caused by air/coating and coating/steel substrate interfaces can be easily determined in the impulse functions and the SWT decomposition coefficients. However, similar to the impulse functions obtained, the SWT approximation coefficients in Fig.5 are smooth curves, from which it is hard to identify the discontinuities, especially when the noise is presented. It can be noted that SWT detail coefficients have amplified the weak information for anticorrosive/ antifouling paint interface. The circles in Fig.5 (c-3) to (e-3) indicated the reflections from antifouling /anticorrosive paint interface. For example, Fig.5 (e-3) was the SWT detail coefficients of the simulated model for three 127um antifouling paint layer plus two $152 \mu \mathrm{m}$ anticorrosive paint layers coated on steel substrate. The circle represented the reflections from antifouling/anticorrosive paint interface and would match the FDTD simulated result collected by the planar sensor of the same model (dotted arrow in the Fig. 4(b-2)). Although they were less obvious than the reflections from the air/coating and coating/steel substrate interfaces, it was enough for interface localization. SWT makes that the existing local features of the data are not suppressed by averaging but observed in their natural position and extension, and it facilitates the identification of salient features in a signal $[44,51-52]$. This would be helpful to distinguish the weak features caused by interfaces between two different mediums with small difference of their RIs.

With the knowledge of refractive index of coating materials, the angle of incidence, and the optical delay between two interfaces, the layer thickness of antifouling and anticorrosive layers can be characterized. When the terahertz wave is perpendicularly incident to the coated sample, the paint layer thickness can be obtained by using the equation as

$$
d_{k}=c \Delta t\left(l_{k}-l_{k-1}\right) / 2 n_{\text {paint }}
$$


where $d_{k}$ represents the paint thickness of measured layer $(k=1,2,3 \ldots$, the case of $k=1$ refers to the first paint layer coated on the steel substrate); $\Delta t$ is time step of simulation; $l_{\mathrm{k}}$ is the interval of two peaks representing the air/coating interface and coating/steel interface in the SWT detail coefficients; $c$ is the speed of light in a vacuum and $n_{\text {paint }}$ is the group refractive index of the measured paint layer. Hence, the proposed method provides the solution for coating thickness analysis of marine protective coatings. 
(a-1)

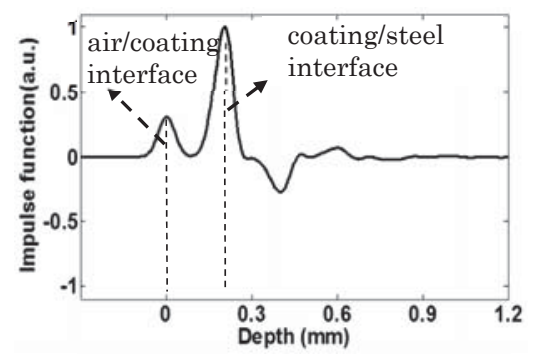

(b-1)

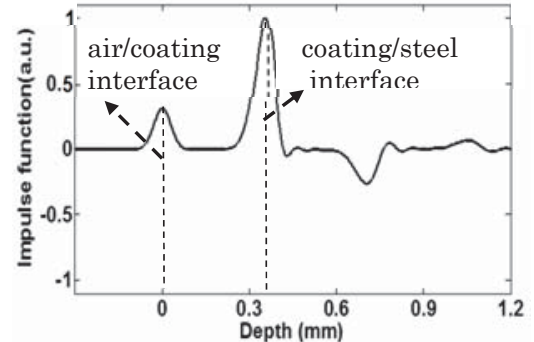

$(c-1)$

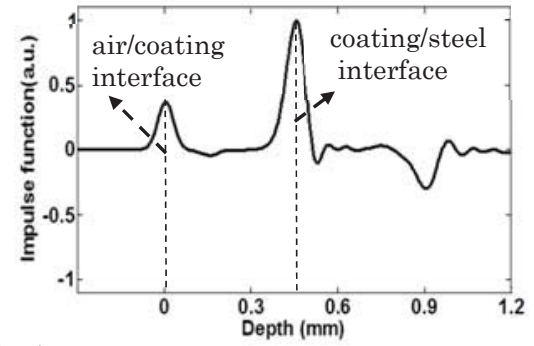

$(d-1)$

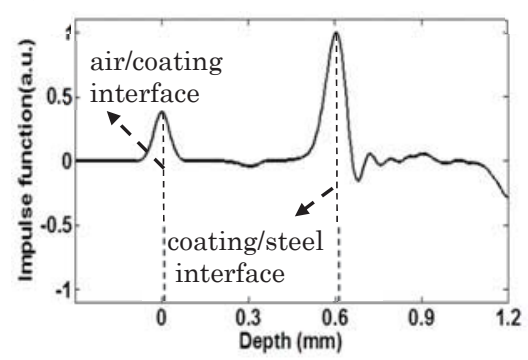

$\left(e^{-1)}\right.$

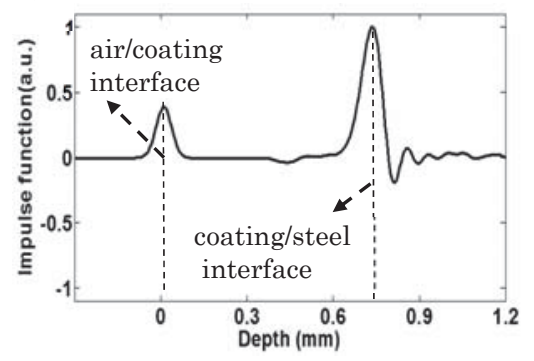

(a-2)

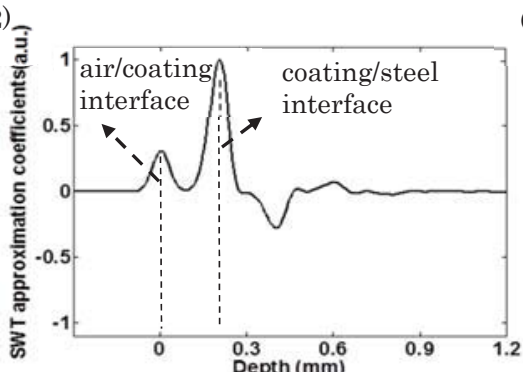

(b-2)

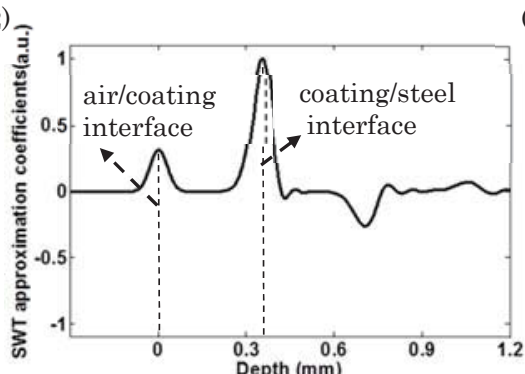

$(c-2)$

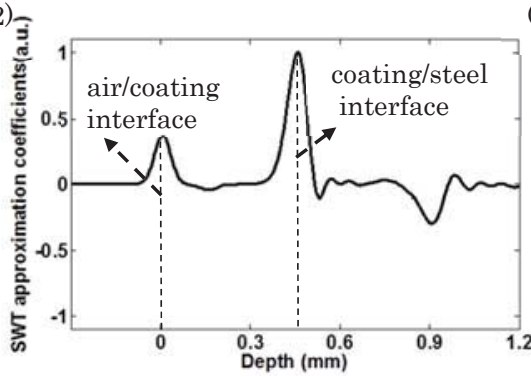

$(\mathrm{d}-2)$

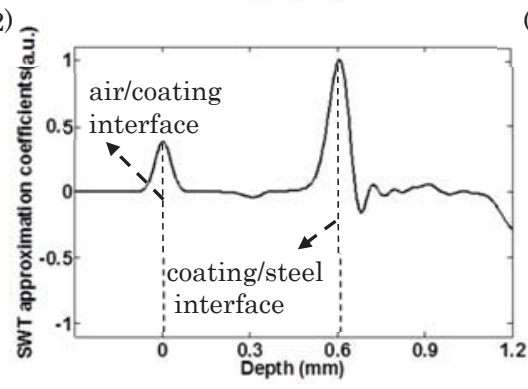

$(\mathrm{e}-2)$

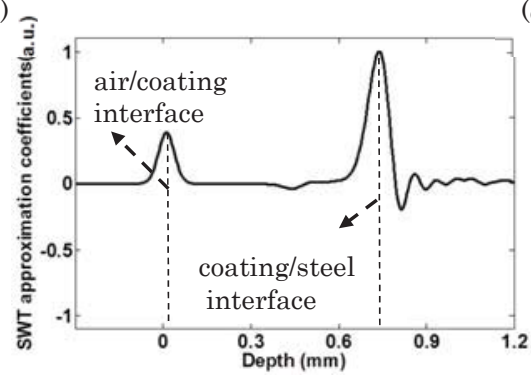

$(\mathrm{a}-3)$

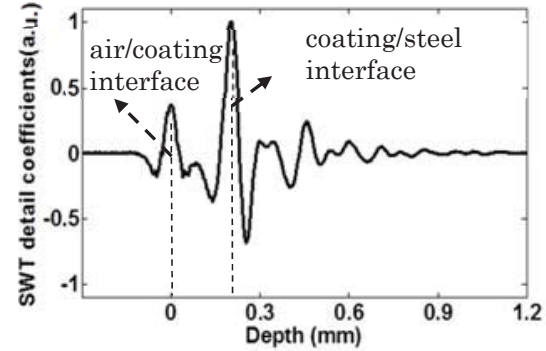

$(b-3)$

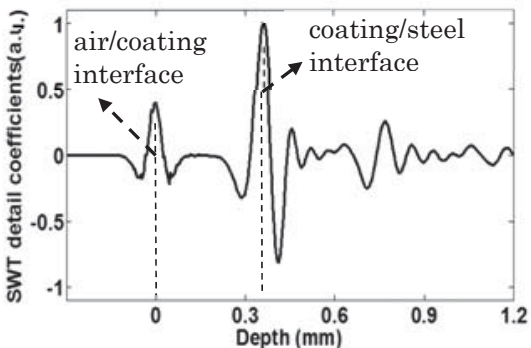

$(c-3)$

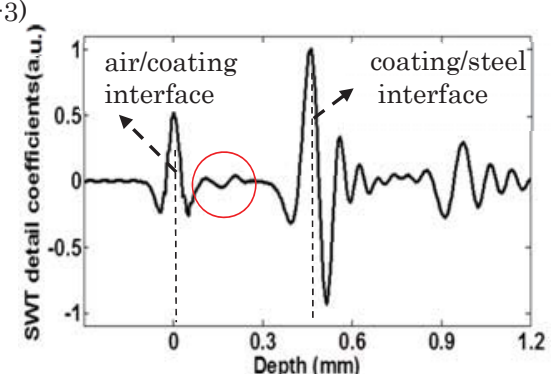

$(\mathrm{d}-3)$

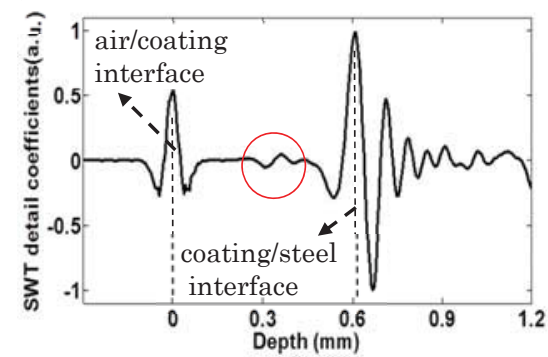

$(\mathrm{e}-3)$

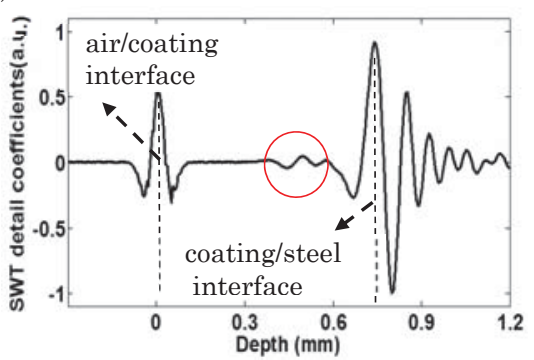

Fig. 5. Impulse functions (left column), SWT appproximation coefficients (middle column) and SWT detail coefficients (right column) of different coated samples. (a) one $152 \mu \mathrm{m}$ anticorrosive paint layers coated on steel substrate; (b) two $152 \mu \mathrm{m}$ anticorrosive paint layers coated on steel substrate; (c) one 127um antifouling paint layer plus two $152 \mu \mathrm{m}$ anticorrosive paint 
layers coated on steel substrate; (d) two $127 \mathrm{um}$ antifouling paint layer plus two $152 \mu \mathrm{m}$ anticorrosive paint layers coated on steel substrate; (e) three $127 \mathrm{um}$ antifouling paint layer plus two $152 \mu \mathrm{m}$ anticorrosive paint layers coated on steel substrate. Circle represented the reflection from anticorrosive/ antifouling paint interface.

The detected data obtained by FDTD method was processed with the proposed algorithm to extract the measured thickness. Table 1 showed the relative error obtained from the theoretical thickness and measured thickness, as mentioned above the value of theoretical thickness and refractive index of paint were obtained from reference [33]. As can be seen from Table 1, most the measured results of paint layer thickness were agreed well with the theoretical values. The maximum relative error was less than $9.25 \%$ ( $4^{\text {th }}$ layer). The errors may be caused by the deconvolution process [51], which decreases in accuracy aspect due to the oversmooth processed signal.

Table 1 Coating thickness of each paint layer of marine protective coatings with three antifouling paint layers and two anticorrosive paint layers

\begin{tabular}{ccccc}
\hline $\begin{array}{c}\text { No. of layer } \\
(k)\end{array}$ & $\begin{array}{c}\text { Refractive } \\
\text { index }\end{array}$ & $\begin{array}{c}\text { Theoretical } \\
\text { thickness } \\
(\text { um })\end{array}$ & $\begin{array}{c}\text { Measured } \\
\text { thickness } \\
(\text { um })\end{array}$ & $\begin{array}{c}\text { Relative } \\
\text { error } \\
(\%)\end{array}$ \\
\hline $1^{\text {st }}$ & 1.74 & 152 & 162 & 6.57 \\
$2^{\text {nd }}$ & 1.74 & 152 & 154.09 & 1.38 \\
$3^{\text {rd }}$ & 1.87 & 127 & 124.88 & 1.67 \\
$4^{\text {th }}$ & 1.87 & 127 & 138.75 & 9.25 \\
$5^{\text {th }}$ & 1.87 & 127 & 124.88 & 1.67 \\
\hline
\end{tabular}

It should be noted that, in the real application of TPI testing of protective coatings, several sources of random and systematic errors exist throughout the measurement process, which can affect the accuracy and uncertainty of testing method. These sources are, for instance, signal noise, sample misalignment, thickness measurement variation, etc [53]. Thus, the added Gaussian with noise in the simulated result could not accurately represent the actual interference in practice. Howevrer, it was often adopted for theoretical studies of THz test method[47]. 


\subsection{Nondestructive Evaluation of Defects beneath Marine Protective Coatings}

In the present work, we also model the occurrence of defects in the marine protective coating, especially the defect beneath the coating surfaces. One of the common defects is the paint-off, which is the detachment of the paint from the substrate or between the different layers of paint and is caused by poor painting quality, contamination on the painting substrate, or deterioration by aging. The second kind is corrosion defect between the coating and substrate, probably caused by the residue of substrate before spraying, or the longterm existence of seawater chemical corrosion through the porosity of coating. There is considerable need for nondestructive inspection of the paint-off area and corrosion since it is very difficult to detect with the conventional thickness meter. Both defects will cause either chemical or structural changes in medium and therefore cause changes in refractive index or absorbing coefficient of the material and can be detected by terahertz radiation technology.

The paint-off defects existing between the different layers of paints were modeled. Two defects with curved shape were simulated by FDTD: 1) one defect with radius of $12 \mathrm{~mm}$ and thickness of $0.18 \mathrm{~mm}$ was embedded inside the three antifouling paint layers, which was located at a distance of $0.074 \mathrm{~mm}$ from the coating surface; 2) the other defect with radius of $12 \mathrm{~mm}$ and thickness of $0.18 \mathrm{~mm}$ was embedded inside the two anticorrosive paint layers, which was located at a distance of $0.461 \mathrm{~mm}$ from the coating surface. The corresponding thickness profiles (Fig.6 (a-1) (b-1)) and FDTD simulation results (Fig.6 (a-2) (b-2)) collected by the planar sensor were shown. From the figure, it can be clearly seen that all the paint layer interfaces and the defects can be identified. Besides, the curved shape of the defect can be also easily distinguished and its location within the coating can be determined. 
$(\mathrm{a}-1)$

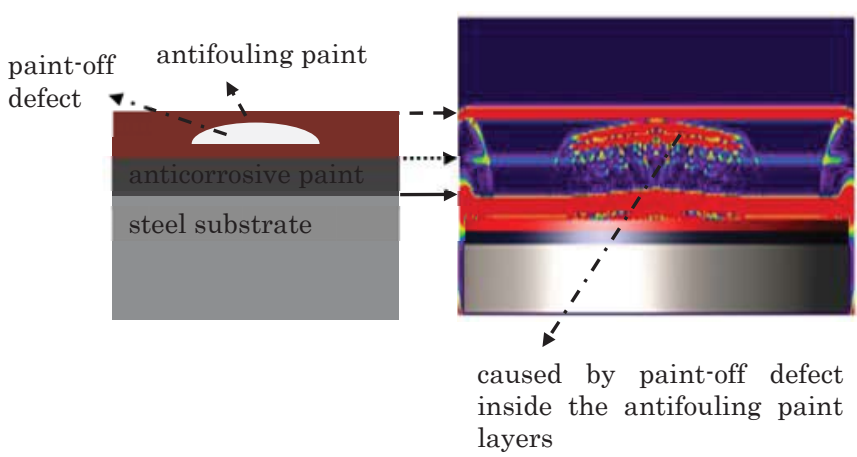

$(\mathrm{b}-1)$

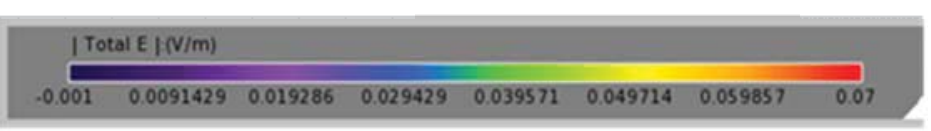

Fig. 6. The thickness profiles $((a-1) \sim(b-1))$ and FDTD simulation results $((a-2) \sim(b-2))$ collected by the planar sensor for marine protective coating with different location of paint-off defect. (a) a defect with radius of $12 \mathrm{~mm}$ and thickness of $0.18 \mathrm{~mm}$ was embedded inside the three antifouling paint layers; (b) a defect with radius of $12 \mathrm{~mm}$ and thickness of $0.18 \mathrm{~mm}$ was embedded inside the anticorrosive paint layers. The colored regions represent the reflected waveforms caused by the inner interfaces between two different materials of the coating. The color stripe in each figure represents the strength of the reflected signals. Dashed arrows denoted reflected waveform from the air/coating surface; solid arrows denoted reflected waveform from the interface between protective coating and steel substrate; dotted arrows denoted reflected waveform from the interface between antifouling paint and anticorrosive paint; dash dotted arrows denote the reflection from the defects.

Furthermore, a far zone sensor was used to obtain the reflection terahertz raw data from the simulated coated steel substrates with defects. The terahertz raw data were deconvoluted with the reference signal to get the terahertz impulse functions, which were then decomposed by SWT into time-frequency domain. Fig.7 showed the comparisons of SWT detail coefficients of intact marine protective coatings and with paint-off defects, where solid line denoted the SWT detail coefficient for intact coating; dashed line denoted the SWT detail coefficient for coating with paint-off defect. The paint-off defect can be identified and the defect position can be exactly determined due to the advantage of local resolution of SWT approach. As shown in Fig.7(a), the reflection caused by paint-off defect was before the one caused by antifouling/anticorrosive paint layer interface, which means that the paint-off defect was located inside the antifouling paint layers. 
Fig.7(b) shown the reflection caused by paint-off defect was after the one caused by antifouling/anticorrosive paint layer interface. It means that the paint-off defect was located inside the anticorrosive paint layers. The results could match the FDTD simulated result collected by the planar sensor of the same model (as shown in Fig. $6(\mathrm{a}-2) \sim(\mathrm{b}-2)$ ). It showed that the SWT decomposition detail coefficients provide a new methodology which is capable of revealing these hidden interface information and to facility the identification of the presence of defect, which is of significance in the quality control of painting process.
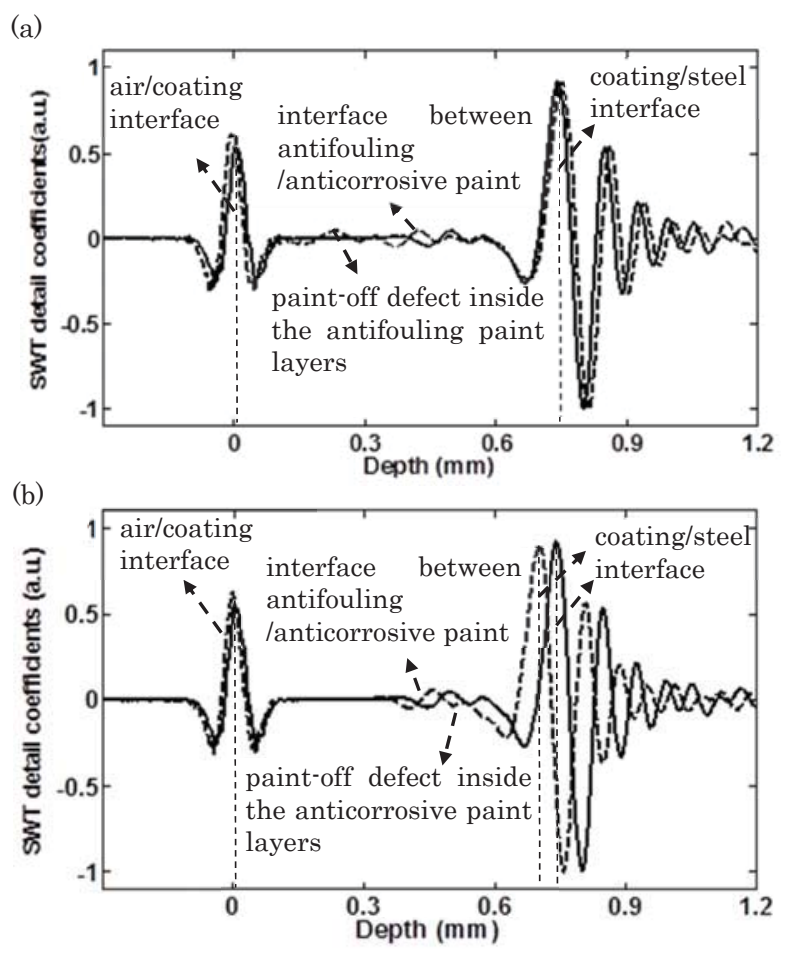

Fig. 7. Comparisons of SWT detail coefficients for intact marine protective coatings and with for coatings paint-off defects. (a) a defect with radius of $12 \mathrm{~mm}$ and thickness of $0.18 \mathrm{~mm}$ was embedded inside the three antifouling paint layers; (b) a defect with radius of $12 \mathrm{~mm}$ and thickness of $0.18 \mathrm{~mm}$ was embedded inside the anticorrosive paint layers. Solid line denoted the SWT detail coefficient for intact coating; dashed line denoted the SWT detail coefficient for coating with paint-off defect.

The investigation about localizing corrosion on the metal substrate, which caused by rust residue before spraying or the long-term existence of seawater chemical corrosion through the porosity of coating, was also made in this paper. For simplicity, a cylinder shape with radius of $12 \mathrm{~mm}$ and thickness of $0.18 \mathrm{~mm}$ 
was taken as corrosion defect to mimic the occurrence of rust residue before spraying. The corrosion defect was set beneath the coatings, whose surface was on the same plane with other part of steel substrate surface. That means that the corrosion medium was implanted between the anticorrosive paint and steel substrate. The material of corrosion defect was set as metal oxide and its refractive index was set as 3.01. The thickness profile and FDTD simulation result collected by the planar sensor for the detected sample with a corrosion defect were shown in Fig. 8 (a-1) and (a-2). Dashed arrow indicated reflected terahertz waveform at the air/coating surface; dotted arrow indicated the reflected waveform at the interface between antifouling paint and anticorrosive paint; solid arrow indicated the reflected waveform at the interface between protective coating and corrosion defect; Dash dot dot arrows indicated the reflected waveform caused by the surface of steel substrate under the corrosion defect. As can be seen from Fig.8 (a-2), due to large differences of RIs of anticorrosive paint and corrosion defect, reflections from anticorrosive paint/corrosion defect interface was strong and obvious. After this strong reflection, there was another clear reflection caused by the surface of steel substrate under the corrosion defect.

Fig.8 (b) showed the corresponding SWT detail coefficients comparison of marine protective coating with and without corrosion defect. Solid line denoted the SWT detail coefficient for intact coating and dashed line denoted the SWT detail coefficient for coating with paint-off defect. From the figure, it can be clearly seen that all the reflection caused by paint layer interfaces. Compared to the sample without a corrosion defect, the maximum peak in the waveform of sample with corrosion defect represented the reflection from anticorrosion paint/corrosion defect interface. The peak was in the same position with the one representing the reflection caused by surface of steel substrate of sample without corrosion defect, because the corrosion defect surface was on the same plane with other part of steel substrate surface of the sample. After this strongest peak, another peak with smaller amplitude caused by the surface of steel substrate can be clearly identified, which match the FDTD simulated result collected by the planar sensor of the same model (Fig. 8 (a-2)). Besides, the strength of this reflection in sample with corrosion defect was 
smaller because corrosion defect had reflected off lot of energy. When compared with the results of coating with paint-off defect, such as Fig.7(a), it was worthy to notice that the reflection strength of corrosion defect was larger than that of paint-off defect, which could be helpful to distinguish the two different kinds of defect, along with the defect formation reason and location. On the other hand, the spatial resolution of conventional terahertz imaging systems is given by the finite spot size of the terahertz focus and limited by the wavelength of the $\mathrm{THz}$ radiation $(0.3 \mathrm{~mm}$ for $1 \mathrm{THz})$ [54]. This is enough for the application of marine protective coating detecting, regarding to the size of defect beneath the coating.

Therefore, the structural change caused by paint-off defect or corrosion defect in the marine protective coating can be detected and analyzed using terahertz wave with stationary wavelet transform. Compared to the expensive Terahertz pulsed imaging (TPI) measurement, FDTD simulation could be cheaper and more flexible to mimic terahertz signals from more multilayered samples. Using the FDTD simulation data of the reflected TPI system, we verified the application of deconvolution combined with SWT approach to improve the analysis precision, speed and also reliability. Using this approach it was possible to estimate the average coating thickness of every layer of paint coated on the steel substrate based on the reflection signal. Furthermore, it was able to provide much more detail information on the position of the defect beneath the coating, such as detachment of large areas where adhesion has failed, and corrosion beneath the coating. Therefore, the combined use of our FDTD simulation algorithms and THz imaging measurements will provide a powerful method for quantitative and non-destructive evaluation of marine protective coatings. Consequently, there is a need for fast data processing algorithms which extract a maximum amount of information out of the measured data in real time. As can be seen from Fig.4, our 3D FDTD model will make it possible to extract useful information from the THz signal measured at the edge of the sample thus allows the TPI map to cover the entire surface of a sample. 

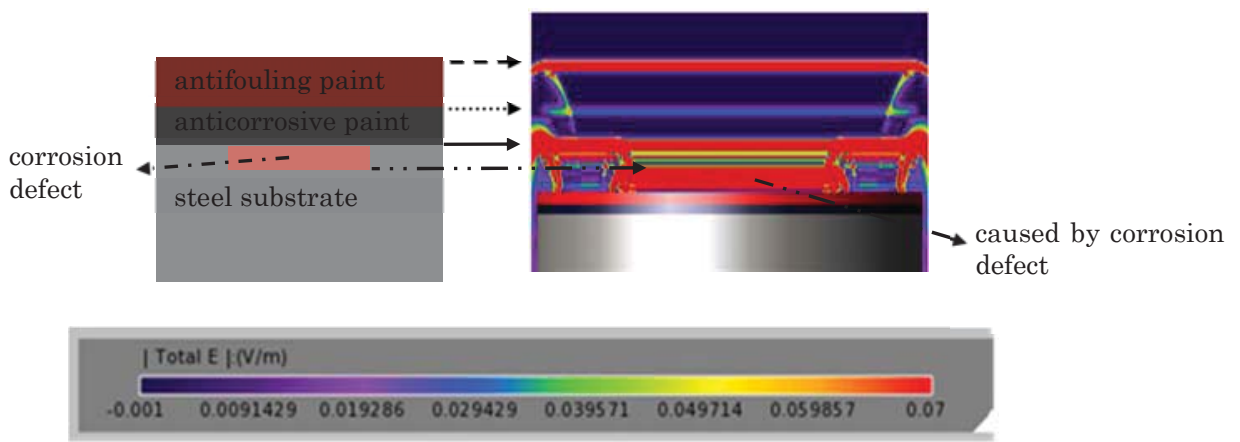

(b)

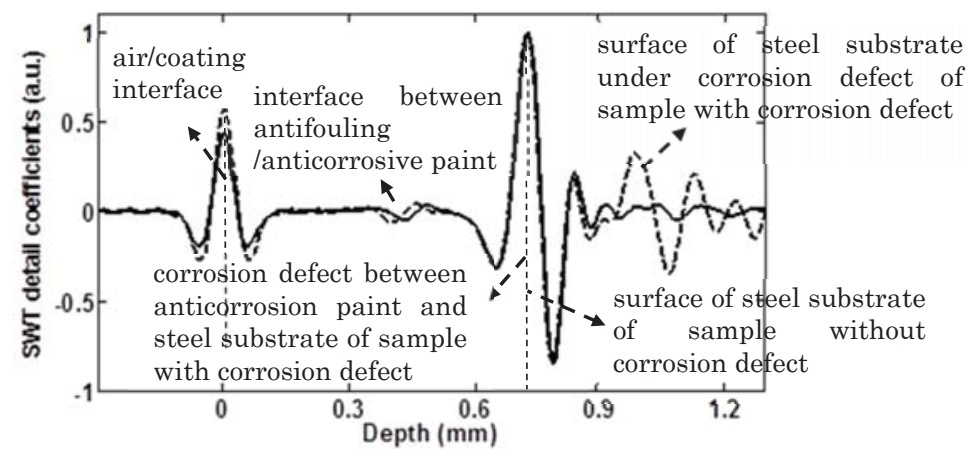

Fig. 8. The thickness profiles (a-1) and FDTD simulation results (a-2) for a marine protective coating model with a corrosion defect, which was collected by the planar sensor: The colored regions represent the reflected waveforms caused by the inner interfaces between two different materials of the coating. The color stripe in each figure represents the strength of the reflected signals. Dashed arrow indicated reflected terahertz waveform at the air/coating surface; dotted arrow indicated the reflected waveform at the interface between antifouling paint and anticorrosive paint; solid arrow indicated the reflected waveform at the interface between protective coating and crossion defect; Dash dot dot arrows indicated the reflected waveform caused by the surface of steel substrate under the corrosion defect. (b) Comparison of the SWT detail coefficients for marine protective coating with and without metal oxide defect. Solid line denoted SWT detail coefficient for coating without defect; dashed line denoted SWT detail coefficient for marine protective coating model with a corrosion defect on the coating/steel interface, which is corresponding to (a-1).

\section{Conclusions}

In summary, a FDTD-based three-dimensional (3D) model was developed to simulate the reflected $\mathrm{THz}$ wave from marine protective coatings and a SWT-based algorithm was employed to characterize the thickness of coating layers and to evaluate the existence of defects. Terahertz impulse function obtained 
from the raw reflected terahertz radiation recorded, is decomposed by stationary wavelet transform into approximation and detail coefficients. The SWT detail coefficient of terahertz impulse function has capability in thickness coating analysis and defect detection of marine protective coating. The proposed method would benefit the effective maintenance to avoid coating failure and facilitate marine protective coating design. Therefore, non-destructive testing and evaluation of marine protective coating system by terahertz waves with stationary wavelet transform could be recommended for engineering applications. We believe that the combined use of the FDTD simulation model with the proposed processing algorithms and THz imaging measurements will provide a powerful method for quantitative and non-destructive evaluation of marine protective coating.

\section{Acknowledgements}

We gratefully acknowledge support from the Fujian Provincial Excellent Young Scientist Fund (2014J07007), Chancellor's Fellowship of University of Strathclyde, the National Natural Science Foundation of China (51005077), Education Department of Fujian Province (JA13180), the Training Program of Fujian Excellent Talents in Universities, the Specialised Research Fund for the Doctoral Program of Higher Education, the Ministry of Education, P. R. China (20133514110008) and the Ministry of Health, P.R. China (WKJ-FJ-27).

\section{References}

1 Soares, C. G., Garbatov, Y., Zayed, A., Wang, G., 2009. Influence of environmental factors on corrosion of ship structures in marine atmosphere. Corros. Sci., 51: 2014-2026.

2 Khobaib, M., Rensi, A., Matakis, T., Donley, M. S., 2001.Real time mapping of corrosion activity under coatings. Prog. Org. Coat. 41: 266-272.

3 Sørensen, P. A., Kiil, S., Dam-Johansen, K., Weinell, C. E., 2009.Anticorrosive coatings: a review. J. Coat. Technol. Res., 6(2) $135-176$.

4 Akpan, U. O., Koko, T. S., Ayyub, B., Dunbar, T. E., 2002.Risk assessment of aging ship hull structures in the presence of corrosion and fatigue. Mar. Struc., 15: 211-231. 
5 Deardorff, J. R., 2009. Non-destructive testing for protective coatings-Implementing a lifetime corrosion prevention program. Met. Finish., 107: 31-39.

6 Heyer, A., D’Souza, F., Zhang, X., Ferrari, G., Mol, J. M. C., de Wit, J. H. W., 2014. Biodegradation of ballast tank coating investigated by impedance spectroscopy and microscopy. Biodegr. , 25:67-83.

7 Láng, G. G., Ujvári, M., Bazsó, F., Vesztergom, S., Ujhelyi, F., 2012. In situ monitoring of the electrochemical degradation of polymer films on metals using the bending beam method and impedance spectroscopy. Electrochim. Acta,73: 59-69.

8 Mansfeld, F., Han, L. T., Lee, C. C., Zhang, G., 1998. Evaluation of corrosion protection by polymer coatings using electrochemical impedance spectroscopy and noise analysis. Electrochim. Acta, 43, pp. 2933-2945.

9 Moshrefi, R., Ghassem Mahjani, M., Ehsani, A., Jafarian, M., 2011. A study of the galvanic corrosion of titanium/L316 stainless steel in artificial seawater using electrochemical noise (EN) measurements andelectrochemical impedance spectroscopy (EIS). Anti-Corros. Method Mater..,58(5): 250- 257.

10 Rodriguez, C. A. G., Aguilar, G. G., Sanchez, A. T. V., Mejía, H. C., 2013. Evaluation of the Degree of Sensitization of a Stainless Steel 304 Using Local Electrochemical Impedance Spectroscopy. ECS Trans., 47(1): 123-129.

11 Aballe, A., Bethencourt, M., Botana, F. J., and Marcos, M., 1999. Using wavelets transform in the analysis of electrochemical noise data. Electrochim. Acta, 44: 4805 - 4816.

12 Mabbutt, S., Mills, D. J., Woodcock, C. P., 2007. Developments of the electrochemical noise method (ENM) for more practical assessment of anti-corrosion coatings. Prog. Org. Coat. 59: 192-196.

13 Zhang, X., Augereau, F., Laux, D., Le Clezio, E., Ismaili, N. A., Kuntz, M., Despaux, G., 2014. Non-destructive Testing of Paint Coatings on Steel Plates by Ultrasonic Reflectometry. Journal of Nondestructive Evaluation, 33(4): 504-514.

14 He, Y. Z., Tian, G. Y., Zhang, H., Alamin, M., Simm, A., Jackson, P., 2012. Steel corrosion characterization using pulsed eddy current systems. Sensors Journal, IEEE, 12(6): 2113-2120.

15 He, Y. Z., Tian, G. Y., Pan, M. C., Chen, D. X., Zhang, H., 2014. An investigation into eddy current pulsed thermography for detection of corrosion blister. Corrosion Science, 78: 1-6.

16 Sfarra, S., Theodorakeas, P., Ibarra-Castanedo, C., Avdelidis, N.P., Paoletti, A., Paoletti, D., Hrissagis, K., Bendada, A., Koui, M., Maldague, X., 2012. Evaluation of defects in panel paintings using infrared, optical and ultrasonic techniques. Insight-NonDestructive Testing and Condition Monitoring, 54(1): 21-27.

17 Yun-Shik, L. 2008. Principles of Terahertz Science and Technology. Springer-Verlag, New York, pp. 159-170.

18 Chantler, C. T., Tran, C. Q., Paterson, D., Cookson, D., Barnea, Z., 2001. X-ray extended-range technique for precision measurement of the X-ray mass attenuation coefficient and Im (f) for copper using synchrotron radiation. Physics Letters A, 
286(5): 338-346.

19 Zhong, S. C., Shen, Y. C., Ho, L., May, R. K., Zeitler, J. A., Evans, M., Taday, P. F., Pepper, M., Rades, T. Gordon, K. C., Muller, R., Kleinebudde, P., 2011. Nondestructive quantification of pharmaceutical tablet coatings using terahertz pulsed imaging and optical coherence tomography. Opt. Laser Eng, 49(3): 361-365.

20 Crawley, D., Longbottom, C., Wallace, V. P., Cole, B., Arnone, D., Pepper, M., 2003. Three-dimensional terahertz pulse imaging of dental tissue. J. Biomed. Opt., 8(2): 303-307.

21 Stoik, C., Bohn, M., Blackshire, J., 2010. Nondestructive evaluation of aircraft composites using reflective terahertz time domain spectroscopy. NDT\&E Int., 43: 106-115.

22 Geltner, I., Hashimshony, D., Zigler, A., 2002. Detection and electrical characterization of hidden layers using time-domain analysis of terahertz reflections. J. Appl. Phys. 92, 203-206.

23 Anastasi, R. F., Madaras, E. I., 2005. Terahertz NDE for under paint corrosion detection and evaluation. AIP Conf. Proc.820,49-56.

24 Zimdars, D., Valdmanis, J. A., White, J. S., Stuk, G., Williamson, S., Winfree, W. P., Madaras, E. I., 2005. Technology and applications of terahertz imaging non - destructive examination: inspection of space shuttle sprayed on foam insulation. AIP Conf. Proc. $760,570-577$.

25 Lopato, P., Chady, T., 2013. Terahertz detection and identification of defects in layered polymer composites and composite coatings. Nondestruct. Test. Eva., 28(1), 28-43.

26 Zhong, H., Xu, J., Xie, X., Yuan, T., Reightler, R., Madaras, E., Zhang, X. C., 2005. Nondestructive defect identification with terahertz time-of-flight tomography. IEEE Sens. J. 5, 203-208.

27 Jackson, J. B., Labaune, J., Mourou, G. A., D'Alessandro, L., Whyte, A., Menu, M., 2011. Pulsed terahertz investigation of corroded and mineralized copper alloy historical artifacts," in 36th International Conference on Infrared, Millimeter and Terahertz Waves (IRMMW-THz), pp. 1-2.

28 Yasui, T., Yasuda, T., Sawanaka, K. I., Araki, T., 2005. Terahertz paintmeter for noncontact monitoring of thickness and drying progress in paint film. Appl. Opt.,44, 6849-6856.

29 Izutani, Y., Akagi, M., Kitagishi, K., 2012. Measurements of Paint Thickness of Automobiles by using THz Time-Domain Spectroscopy 37th International Conference on Infrared, Millimeter, and Terahertz Waves (IRMMW-THz), , pp. 1-2.

30 Fukuchi, T., Fuse, N., Okada, M., Fujii, T., Mizuno, M., Fukunaga, K., 2013. Measurement of Refractive Index and Thickness of Topcoat of Thermal Barrier Coating by Reflection Measurement of Terahertz Waves. Electron. Comm. Jpn., 96(12), $37-45$. 31 Kurabayashi, T., Yodokawa, S., and Kosaka, S., 2012. Terahertz imaging through paint layers. 37th International Conference 
on Infrared, Millimeter, and Terahertz Waves (IRMMW-THz), pp. 1-2.

32 Cook, D. J., Sharpe, S. J., Lee, S., Allen, M. G., 2007. Terahertz time domain measurements of marine paint thickness. Opt. Terahertz Sci. Tec., TuB5.

33 Cook, D. J., Lee, S., Sharpe, S. J., Allen, M. G., 2008. Accuracy and linearity of time-domain THz paint thickness measurements. Proc. of SPIE Vol. 6893, 68930H.

34 Tirkas, P. A., Balanis, C. A., Purchine, M. P., Barber, G. C., 1993. Finite-difference time-domain method for electromagnetic radiation, interference, and interaction with complex structures. IEEE Trans. Electromag. Compat. 35, $192-203$.

35 Kumar, N. S., Alamelu, N., Bharathy, B. R., Piriyavathani, V., 2008. FDTD based evaluation of Specific Absorption Rate distribution in human head due to mobile phones," 10th International Conference on in Electromagnetic Interference \& Compatibility, pp. 411-417.

36 Larsen, C., Cooke, D. G., Jepsen, P. U., 2011. Finite-difference time domain analysis of time-resolved terahertz spectroscopy experiments. J. Opt. Soc. Am. B28, 1308-1316.

37 Tu, W. L., Zhong, S. C., Yao, H. Z., Shen, Y. C., 2013. FDTD-based computed terahertz wave propagation in multilayer medium structures.. Proc. of SPIE. 2013 International Symposium on Photoelectronic Detection and Imaging: Terahertz Technologies and Applications, 8909: 89090Y1.

38 Tu, W., Zhong, S., Shen, Y., Zhou, Q., Yao, L., 2014. FDTD-based quantitative analysis of terahertz wave detection for multilayered structures. J. Opt. Soc. Am. A, 31: 2285-2293.

39 Taflove, A., Umashankar, K. R., 1990. The finite-difference time-domain method for numerical modeling of electromagnetic wave interactions. Electromag., 10(1-2), 105-126.

40 Korkut, E., Atlar, M., 2012. An experimental investigation of the effect of foul release coating application on performance, noise and cavitation characteristics of marine propellers, Ocean Eng., 41, 1-12.

41 Paik, B. G., Kim, K. S., Kim, K. Y., Ahn, J. W., Kim, T. G., Kim, K. R., Jang, Y. H., Lee, S. U., 2011. Test method of cavitation erosion for marine coatings with low hardness. Ocean Eng., 38(13), 1495-1502.

42 Nason, G. P., Silverman, B. W., 1995. The stationary wavelet transform and some statistical applications. Wavelets and statistics, 281-299. Springer, New York.

43 Nema, M., Gupta, L., Trivedi, N. R., 2012. Video Compression using SPIHT and SWT Wavelet. Int. J. Electron. Comm. Eng. 5(1): $1-8$.

44 Zhong, S., Oyadiji, S. O., 2007. Crack detection in simply supported beams without baseline modal parameters by stationary wavelet transform. Mech. Syst. Signal Pr., 21(4), 1853-1884. 
45 Yasuda, T., Iwata, T., Araki, T., Yasui, T., 2007. Improvement of minimum paint film thickness for THz paint meters by multiple-regression analysis. Applied optics, 46(30): 7518-7526.

46 Johnson, J. L., Dorney, T. D., Mittleman, D.M., 2001. Interferometric imaging with terahertz pulses. IEEE Journal of Selected Topics in Quantum Electronics, 7: 592-599.

47 Chen, Y., Huang, S., Pickwell-MacPherson, E., 2010. Frequency-wavelet domain deconvolution for terahertz reflection imaging and spectroscopy. Opt. Express, 18(2), 1177-1190.

48 Tsai, T. R., Chen, S. J., Chang, C. F., Hsu, S. H., Lin, T. Y., Chi, C. C., 2006. Terahertz response of GaN thin films. Opt. Express, 14(11): 4898-4907.

49 Russe, I. S., Brock, D., Knop, K., Kleinebudde, P., Zeitler, J. A. 2012. Validation of Terahertz Coating Thickness Measurements Using X-ray Microtomography. Mol. Pharm., 9(12): 3551-3559.

50 Pickwell, E., Cole, B. E., Fitzgerald, A. J., Pepper, M., Wallace, V. P., 2004. In vivo study of human skin using pulsed terahertz radiation. Phys. Med. Biol., 49(9), 1595.

51 Zhong, S., Shen, Y. C., Evans, M., Zeitler, J. A., May, R. K., Gladden, L. F., Byers, C., 2009. Quantification of thin-film coating thickness of pharmaceutical tablets using wavelet analysis of terahertz pulsed imaging data. 34th International Conference on Infrared, Millimeter, and Terahertz Waves, pp: 1-2.

52 van Berkel, M., 2010. Wavelet analysis for real-time determination of the sawtooth behavior in non-stationary fusion plasmas. Master's Thesis, Eindhoven University of Technology

53 Withayachumnankul, W., Fischer, B. M., Lin, H., Abbott, D., 2008. Uncertainty in terahertz time-domain spectroscopy measurement. JOSA B, 25(6): 1059-1072.

54 Zhang, X. C., 2002. Terahertz wave imaging: horizons and hurdles. Physics in Medicine and Biology, 47(21): 3667. 